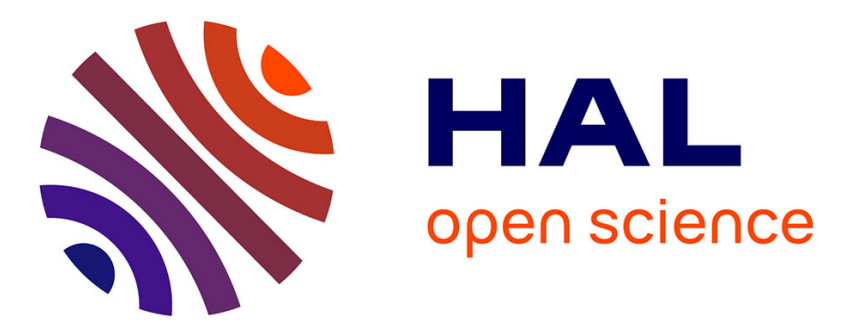

\title{
Non-Gaussian noise benefits for coherent detection of narrowband weak signal
}

Fabing Duan, François Chapeau-Blondeau, Derek Abbott

\section{To cite this version:}

Fabing Duan, François Chapeau-Blondeau, Derek Abbott. Non-Gaussian noise benefits for coherent detection of narrowband weak signal. Physics Letters A, 2014, 378 (26-27), pp.1820-1824. 10.1016/j.physleta.2014.04.061 . hal-02171095

\section{HAL Id: hal-02171095 \\ https://hal.science/hal-02171095}

Submitted on 20 Sep 2021

HAL is a multi-disciplinary open access archive for the deposit and dissemination of scientific research documents, whether they are published or not. The documents may come from teaching and research institutions in France or abroad, or from public or private research centers.
L'archive ouverte pluridisciplinaire HAL, est destinée au dépôt et à la diffusion de documents scientifiques de niveau recherche, publiés ou non, émanant des établissements d'enseignement et de recherche français ou étrangers, des laboratoires publics ou privés.

\section{(c)(1)}

Distributed under a Creative Commons Attribution| 4.0 International License 


\title{
Non-Gaussian noise benefits for coherent detection of narrowband weak signal
}

\author{
Fabing Duan ${ }^{\mathrm{a}, *}$, François Chapeau-Blondeau ${ }^{\mathrm{b}}$, Derek Abbott ${ }^{\mathrm{c}}$ \\ a Institute of Complexity Science, Qingdao University, Qingdao 266071, PR China \\ b Laboratoire Angevin de Recherche en Ingénierie des Systèmes (LARIS), Université d'Angers, 62 avenue Notre Dame du Lac, 49000 Angers, France \\ ' Centre for Biomedical Engineering (CBME) and School of Electrical E' Electronic Engineering, The University of Adelaide, Adelaide, SA 5005, Australia
}

In an ad hoc suboptimal detector, the benefits of non-Gaussian noise to narrowband weak signal detection are demonstrated. Particularly, for a noise envelope with a Rice distribution, we can improve the detector performance by tuning threshold parameter but keeping noise level, or increasing the noise level for a fixed threshold. It is verified that, under certain circumstances, the optimal detection probability achieved by tuning noise level is superior to that obtained by optimizing the detector threshold.

\section{Introduction}

In long-range communication and radar systems, the receiver bandwidth is sufficiently narrow that only the frequency components centered on a carrier frequency can pass. In this context, narrowband noise refers to possessing a bandwidth that is sufficiently narrow relative to the carrier frequency [1-4]. For detecting weak narrowband signals, the generalized Neyman-Pearson criterion leads to an asymptotic locally optimum detector on the basis of a known noise distribution [1-4]. When a priori knowledge of noise is absent or the noise intensity is time-varying, a number of ad hoc correlation detectors can be practically employed, which can provide comparable robust performance relative to the locally optimum detector in a variety of underlying noise environments [1-4]. Although an ad hoc detector is suboptimal to the corresponding locally optimum one, recent research results show that there is an opportunity for exploiting noise benefit, i.e. stochastic resonance [5-19]. The essential feature of stochastic resonance is the performance enhancement of nonlinear systems by an appropriate non-zero noise level [5-19]. In the field of signal detection, noiseenhanced low-pass known signal detection has been frequently reported $[6-15,20-22]$. In these studies, the low-pass signal has a power spectrum concentrated around zero, and its bandwidth is

\footnotetext{
* Corresponding author.

E-mail addresses: fabing.duan@gmail.com (F. Duan), chapeau@univ-angers.fr (F. Chapeau-Blondeau), dabbott@adelaide.edu.au (D. Abbott).
}

much smaller than the cut-off frequency. Noise is assumed to have a wide frequency band, i.e. broadband noise. It is clearly shown [9-15,20-22] that noise, as an optional approach, can improve the detection performance of suboptimal but practical nonlinear detectors.

In this letter, we focus on the benefits of non-Gaussian noise to narrowband weak signal detection in an ad hoc hard-limiter correlation detector. Three noise envelope distributions, of generalized Rayleigh, Hall and Rice models, are observed to possess the potential ability of improving the detector performance. In particular, for the Rice distribution model of noise envelope, the detector performance can be enhanced by two methods. One is by tuning threshold parameter, while the noise level is fixed. The other way is by increasing noise level for a fixed threshold parameter. We prove that the maximum detection probability achieved by tuning noise level is superior to that obtained by tuning the detector threshold. This advantageous result further confirms the potential capability of noise in performance improvement in the context of nonlinear signal processing.

\section{Narrowband signal detection model}

Consider the observation model of a known narrowband signal observed in additive noise [1-4]

$X(t)=\theta v(t) \cos \left[2 \pi f_{0} t+\phi(t)\right]+W(t)$, 
where $v(t)$ and $\phi(t)$ are known amplitude and phase modulations, and $\theta$ is the overall signal strength. The carrier frequency is $f_{0}$, and the noise process $W(t)$ is assumed to be stationary, zero-mean, bandlimited white noise with a constant power spectral density over a frequency band $\left(f_{0}-B / 2, f_{0}+B / 2\right)$ and zero outside $\left(B \ll f_{0}\right)$. The narrowband noise process $W(t)$ can be expressed as [1]

$$
W(t)=W_{I}(t) \cos \left(2 \pi f_{0} t\right)+W_{Q}(t) \sin \left(2 \pi f_{0} t\right)
$$

with its inphase and quadrature components $W_{I}(t)$ and $W_{Q}(t)$, respectively. Assume $s_{I}(t)=v(t) \cos \phi(t)$ and $s_{Q}(t)=-v(t) \sin \phi(t)$, the observation model of Eq. (1) can be also represented as

$$
X(t)=X_{I}(t) \cos \left(2 \pi f_{0} t\right)+X_{Q}(t) \sin \left(2 \pi f_{0} t\right)
$$

with the inphase component $X_{I}(t)=\theta s_{I}(t)+W_{I}(t)$ and the quadrature component $X_{Q}(t)=\theta s_{Q}(t)+W_{Q}(t)$. Given a set of samples of observation $X_{i}, i=1,2, \cdots, n$, we are interested of testing $\theta=0$ versus $\theta>0$, formulated by binary hypotheses on the joint probability density function

$$
\begin{aligned}
& H_{0}: \quad f_{I Q}\left(X_{I}, X_{Q}\right)=\prod_{i=1}^{n} f_{I Q}\left(X_{I i}, X_{Q i}\right), \quad \text { for } \theta=0, \\
& H_{1}: \quad f_{I Q}\left(X_{I}, X_{Q}\right)=\prod_{i=1}^{n} f_{I Q}\left(X_{I i}-\theta s_{I i}, X_{Q i}-\theta s_{Q i}\right),
\end{aligned}
$$

for $\theta>0$,

where the inphase and quadrature samples $W_{I i}$ and $W_{Q i}$ form a set of statistical independent random vectors governed by the common bivariate joint probability density function $f_{I Q}[1-4]$.

Based on generalized Neyman-Pearson criterion [1-4], the locally optimum detector takes a test statistic $T_{\mathrm{LO}}$ representing the maximum derivative of the logarithm of the joint probability density function $f_{I Q}$ at $\theta=0$. Therefore, the locally optimum test statistic $T_{\mathrm{LO}}$ is given by [1-4]

$$
\begin{aligned}
T_{\mathrm{LO}}\left(X_{I}, X_{Q}\right)= & \left.\frac{d}{d \theta}\left[\ln \prod_{i=1}^{n} f_{I Q}\left(X_{I i}-\theta s_{I i}, X_{Q i}-\theta s_{Q i}\right)\right]\right|_{\theta=0} \\
\approx & \sum_{i=1}^{n} s_{I i}\left[\frac{-\frac{\partial}{\partial X_{I i}} f_{I Q}\left(X_{I i}, X_{Q i}\right)}{f_{I Q}\left(X_{I i}, X_{Q i}\right)}\right] \\
& +\sum_{i=1}^{n} s_{Q i}\left[\frac{-\frac{\partial}{\partial X_{Q i}} f_{I Q}\left(X_{I i}, X_{Q i}\right)}{f_{I Q}\left(X_{I i}, X_{Q i}\right)}\right] .
\end{aligned}
$$

Furthermore, assume the observation envelope $R_{i}=\sqrt{X_{I i}^{2}+X_{Q i}^{2}}$ and phase variable $\Phi_{i}=\arctan \left(X_{I i} / X_{Q i}\right)$ at the $i$-th sampling time. An interesting noise model is with a circularly symmetric bivariate density function under the hypothesis $H_{0}$, i.e. $f_{I Q}\left(x_{I}, x_{Q}\right)=$ $h(R)$. Then, the noise envelope distribution can be expressed as $f_{R}(R)=2 \pi R h(R)$, and the phase $\Phi$ is uniformly distributed over $[0,2 \pi]$ with $f_{\Phi}(\Phi)=1 /(2 \pi)$. With circular symmetry, the locally optimum test statistic $T_{\text {LO }}$ of Eq. (6) becomes

$$
\begin{aligned}
T_{\mathrm{LO}}\left(X_{I}, X_{Q}\right) & =\sum_{i=1}^{n}-s_{I i} \frac{X_{I i}}{R_{i}} \frac{h^{\prime}\left(R_{i}\right)}{h\left(R_{i}\right)}-s_{Q i} \frac{X_{Q i}}{R_{i}} \frac{h^{\prime}\left(R_{i}\right)}{h\left(R_{i}\right)} \\
& =\sum_{i=1}^{n} g_{\mathrm{LO}}\left(R_{i}\right)\left[s_{I i} X_{I i}+s_{Q i} X_{Q i}\right],
\end{aligned}
$$

with the derivative $h^{\prime}(R)=d h(R) / d R$ and the locally optimum nonlinearity $g_{\mathrm{LO}}(R)=-h^{\prime}(R) /[R h(R)]$, which is a sufficient statistic under the assumptions of weak signal limit and large sample size $[1-4]$.
However, the structure of the locally optimal nonlinearity $g_{\mathrm{LO}}(R)$ in Eq. (7) is determined by the noise probability distribution and also the noise level. Then, for some practical signal processing tasks, the locally optimal nonlinearity may be too complex to be implemented, and also cannot be established for an unknown noise distribution $[1,4,15]$. Therefore, this provides an opportunity for the suboptimal nonlinearity to improve the detectability by the SR effect. Thus, we consider the test statistic of a generalized narrowband correlation detector given by

$$
\begin{aligned}
T\left(X_{I}, X_{Q}\right) & =\sum_{i=1}^{n} g\left(R_{i}\right)\left(s_{I i} X_{I i}+s_{Q i} X_{Q i}\right) \\
& =\sum_{i=1}^{n} R_{i} g\left(R_{i}\right)\left[s_{I i} \cos \left(\Phi_{i}\right)+s_{Q i} \sin \left(\Phi_{i}\right)\right],
\end{aligned}
$$

where the characteristic $g(R)$ is an ad hoc piecewise nonlinearity being a function of the observation envelope $R[1,4]$. Since the phase $\Phi$ is uniformly distributed over $[0,2 \pi]$, then under the hypothesis $H_{0}$, the expectation

$$
\mathrm{E}\left[T \mid H_{0}\right]=\sum_{i=1}^{n} \mathrm{E}_{R}\left[R_{i} g\left(R_{i}\right)\right] \mathrm{E}_{\Phi}\left[s_{I i} \cos \left(\Phi_{i}\right)+s_{Q i} \sin \left(\Phi_{i}\right)\right]=0
$$

and the variance

$$
\begin{aligned}
\operatorname{var}\left[T \mid H_{0}\right] & =\sum_{i=1}^{n} \mathrm{E}_{R}\left[R_{i}^{2} g^{2}\left(R_{i}\right)\right] \mathrm{E}_{\Phi}\left\{\left[s_{I i} \sin \left(\Phi_{i}\right)+s_{Q i} \cos \left(\Phi_{i}\right)\right]^{2}\right\} \\
& =\mathrm{E}_{R}\left[R^{2} g^{2}(R)\right] \sum_{i=1}^{n}\left(s_{I i}^{2}+s_{Q i}^{2}\right) / 2 \\
& =n P_{S}^{2} \mathrm{E}_{R}\left[R^{2} g^{2}(R)\right],
\end{aligned}
$$

where $\mathrm{E}_{R}[\cdot]=\int_{0}^{\infty} \cdot f_{R} d R, \mathrm{E}_{\Phi}[\cdot]=\int_{0}^{2 \pi} \cdot f_{\Phi} d \Phi$ and the average signal power $P_{s}^{2}=\sum_{i=1}^{n}\left(s_{I i}^{2}+s_{Q i}^{2}\right) /(2 n)$. Under the hypothesis $H_{1}$ and for weak signal strength $\theta \rightarrow 0$, the joint distribution can be expanded to the first-order as

$$
\begin{aligned}
& f_{I Q}\left(x_{I i}-\theta s_{I i}, x_{Q i}-\theta s_{Q i}\right) \\
& \quad \approx h\left(R_{i}\right)-\theta h^{\prime}\left(R_{i}\right)\left[s_{I i} \cos \left(\Phi_{i}\right)+s_{Q i} \sin \left(\Phi_{i}\right)\right] .
\end{aligned}
$$

Then, the expectation $\mathrm{E}\left[T \mid H_{1}\right]$ can be approximated as

$$
\begin{aligned}
\mathrm{E}\left[T \mid H_{1}\right] \approx & -\theta \mathrm{E}_{R}\left[R g(R) h^{\prime}(R) / h(R)\right] \sum_{i=1}^{n} \mathrm{E}_{\Phi}\left\{\left[s_{I i} \sin \left(\Phi_{i}\right)\right.\right. \\
& \left.\left.+s_{Q i} \cos \left(\Phi_{i}\right)\right]^{2}\right\} \\
= & -\theta n P_{S}^{2} \mathrm{E}_{R}\left[\operatorname{Rg}(R) h^{\prime}(R) / h(R)\right],
\end{aligned}
$$

and the variance $\operatorname{var}\left[T \mid H_{1}\right] \approx \operatorname{var}\left[T \mid H_{0}\right][1-4]$.

Therefore, in the asymptotic case of $\theta \rightarrow 0$ and $n \rightarrow \infty$, the test statistics $T$, according to the central limit theorem, both converge to Gaussian distributions under hypothesis $H_{0}$ and $H_{1}$. For a fixed false alarm probability $P_{f}$, the decision threshold $\gamma$ can be determined. When the test statistic $T\left(X_{I i}, X_{Q i}\right)>\gamma$, the hypothesis $H_{1}$ is accepted. Fig. 1 illustrates the structure of the generalized narrowband correlation detector for hypothesis $H_{1}$ versus hypothesis $H_{0}$. Thus, the detection probability $P_{d}[1-4]$ can be computed as

$P_{d}=Q\left[Q^{-1}\left(P_{f}\right)-\sqrt{n \theta^{2} P_{s}^{2}} \sqrt{\xi(T)}\right]$,

with $Q(x)=\int_{x}^{\infty} \exp \left[-t^{2} / 2\right] / \sqrt{2 \pi} d t$ and its inverse function $Q^{-1}(x)$. Here, the total signal power is $n \theta^{2} P_{s}^{2}$ for the observation 


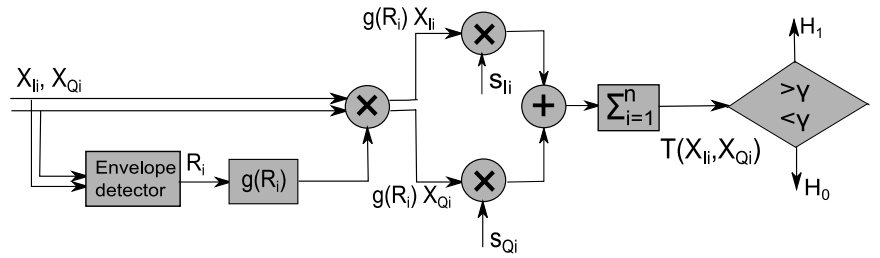

Fig. 1. Structure of generalized narrowband correlation detector.

data with size $n$, and the detection probability $P_{d}$ is a monotonically increasing function of the normalized detector efficacy defined as $[1-4]$

$\xi(T)=\lim _{n \rightarrow \infty} \frac{\left\{d \mathrm{E}\left[T(X) \mid H_{1}\right] /\left.d \theta\right|_{\theta=0}\right\}^{2}}{n P_{s}^{2} \operatorname{var}\left[T(X) \mid H_{0}\right]}=\frac{\mathrm{E}_{R}^{2}\left[R g(R) h^{\prime}(R) / h(R)\right]}{\mathrm{E}_{R}\left[R^{2} g^{2}(R)\right]}$.

\section{Results of noise benefits}

Based on the Schwarz-Cauchy inequality, the efficacy $\xi(T)$ in Eq. (14) is maximized as $\xi_{\max }(T)=\mathrm{E}_{R}\left[h^{\prime 2}(R) / h^{2}(R)\right]$ by the locally optimum nonlinearity $g_{\mathrm{LO}}(R)=C h^{\prime}(R) /[R h(R)]$ for any constant $C$ [1]. It is noted that $g_{\mathrm{LO}}$ is designed on the basis of explicit a priori knowledge of the underlying noise environment, which is sometimes difficult or costly to implement due to its complexity [1-4]. In practice, suboptimal detectors are employed, which comprises the nonlinear characteristic $g(R)$ in Eq. (8) usually chosen as an ad hoc piecewise function. More specifically, here we consider the hard-limiter characteristic

$g_{\lambda}(R)= \begin{cases}1 / R, & R \geq \lambda, \\ 0, & 0 \leq R<\lambda,\end{cases}$

where the threshold parameter $\lambda \geq 0$. Then, the detector efficacy can be computed as

$\xi(T)=\frac{\mathrm{E}_{R}^{2}\left[R g_{\lambda}(R) h^{\prime}(R) / h(R)\right]}{\mathrm{E}_{R}\left[R^{2} g_{\lambda}^{2}(R)\right]}=\frac{\left[f_{R}(\lambda)+\int_{\lambda}^{\infty} f_{R}(R) / R d R\right]^{2}}{1-F_{R}(\lambda)}$,

with the cumulative distribution function $F_{R}(R)=\int_{0}^{R} f_{R}(r) d r$. Substituting Eq. (16) into Eq. (13), we can calculate the detection probability $P_{d}$ for the considered noise envelope density.

We first consider the generalized Rayleigh noise envelope density

$f_{R}(R)=\frac{R \alpha(k)}{\sigma^{2}} \exp \left[-\left(\frac{R}{\sigma \beta(k)}\right)^{k}\right], \quad R \geq 0$,

with $\alpha(k)=k \beta^{-2}(k) \Gamma^{-1}(2 / k)$ and $\beta(k)=\sqrt{2 \Gamma(2 / k) / \Gamma(4 / k)}$ $[1,2,4]$. Here, $\sigma^{2}$ is the common variance of the inphase and quadrature noise components, and $k$ is the exponential parameter. For a fixed threshold parameter $\lambda=1$, the detection probability $P_{d}$ is plotted as a function of noise level $\sigma$ for different exponents $k$, as shown in Fig. 2. It is seen in Fig. 2 that there is an optimal noise level $\sigma$ corresponding to the maximum $P_{d}$. For larger exponents $k$, the maximum detection probability $P_{d}$ is apparently enhanced by noise. This ad hoc hard-limiter characteristic is suitable for detecting weak signals in more impulsive noise with large exponents $k$. Here, the false alarm probability is a constant $P_{f}=10^{-3}$. For a large observation size $n$ and the overall signal strength $\theta$, the average signal power $P_{s}^{2}$ is chosen in such a way that the total signal power $n \theta^{2} P_{s}^{2}=1$ in Eq. (13).

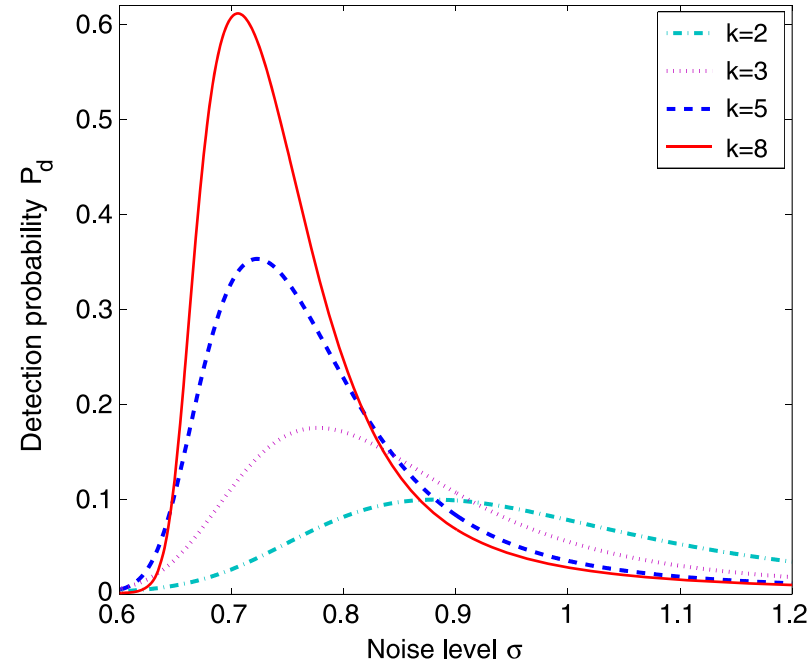

Fig. 2. Detection probability $P_{d}$ as a function of noise level $\sigma$ of generalized Rayleigh model from Eq. (17) for different exponents $k$.

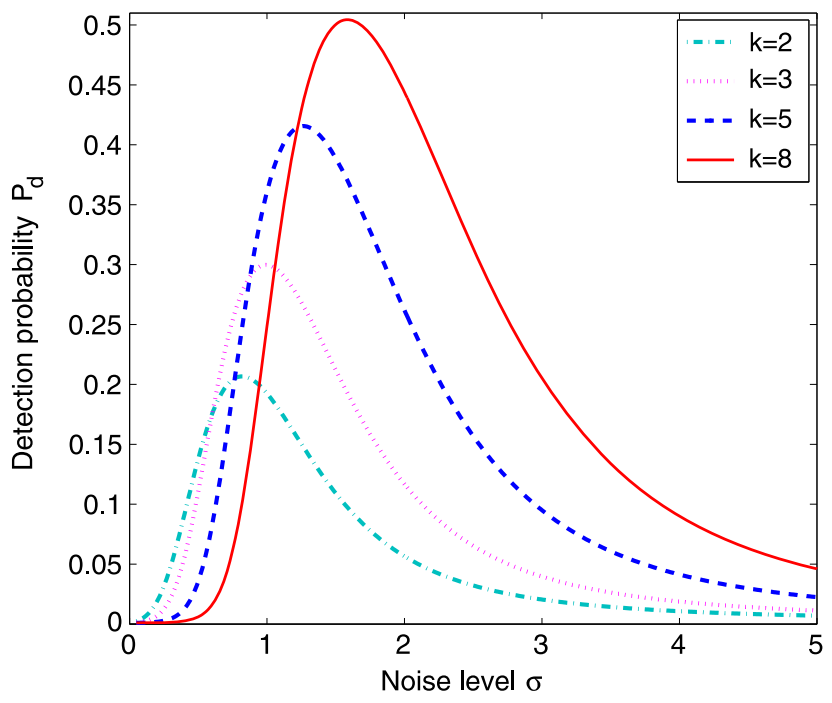

Fig. 3. Detection probability $P_{d}$ as a function of noise level $\sigma$ of Hall model from Eq. (18) for different exponents $k$.

We further consider an atmospheric channel noise of Hall distribution model with its envelope density

$f_{R}(R)=\frac{k R \sigma^{k}}{\left(R^{2}+\sigma^{2}\right)^{1+k / 2}}, \quad R \geq 0$,

where $\sigma$ is the scale parameter and the exponent parameter is $k$ [23]. For a given $k$, the scale parameter $\sigma$ represents the ratio of Gaussian noise variance to the variance of slowly modulation process [23]. It is shown in Fig. 3 that, upon increasing the noise scale parameter $\sigma$, the detection probability $P_{d}$ also exhibits the noiseenhanced effect for different exponents $k$. In Fig. 3, the threshold parameter $\lambda=1$, the constant false alarm probability $P_{f}=10^{-3}$ and the total signal power $n \theta^{2} P_{s}^{2}=2$. Here, we emphasize that the value of overall signal power $n \theta^{2} P_{s}^{2}$ will affect the detection probability $P_{d}$, but not the occurrence of stochastic resonance.

We finally consider the inphase and quadrature noise components $X_{I}$ and $X_{Q}$ both satisfying the Gaussian mixture noise distribution as

$f(x)=\frac{1}{2 \sqrt{2 \pi \epsilon^{2}}}\left[\exp \left(\frac{-(x-\mu)^{2}}{2 \epsilon^{2}}\right)+\exp \left(\frac{-(x+\mu)^{2}}{2 \epsilon^{2}}\right)\right]$, 


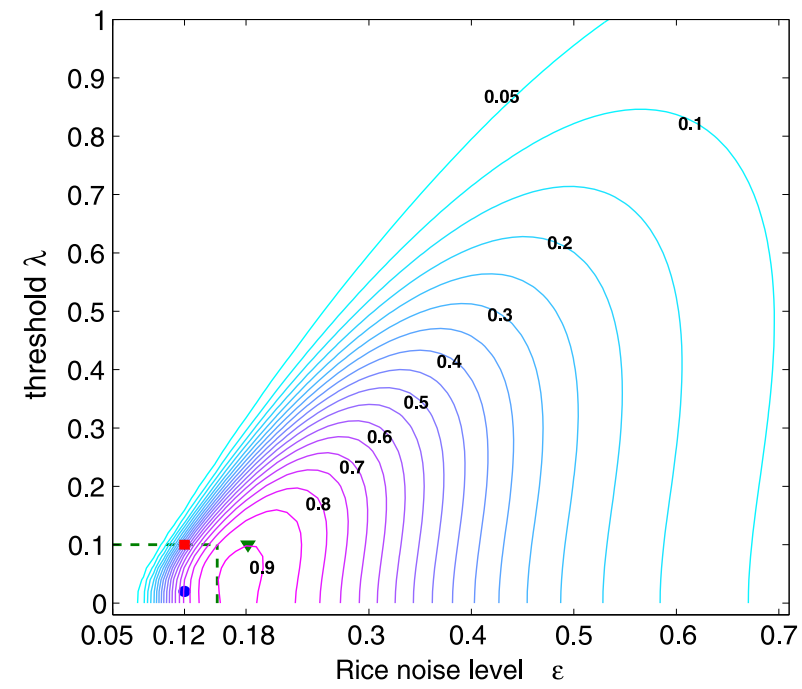

Fig. 4. Contours of detection probability $P_{d}$ as a function of the noise level $\epsilon$ of the Rice model from Eq. (20) and the detector threshold $\lambda$. Here, the parameter $\mu=0.1$ in Eq. (20). (For interpretation of the colors in this figure, the reader is referred to the web version of this article.)

where the variance $\sigma^{2}=\mu^{2}+\epsilon^{2}$ and parameters $\mu, \epsilon \geq 0$. After some mathematical manipulations, the envelope $R=\sqrt{X_{I}^{2}+X_{Q}^{2}}$ can be found to have the Rice distribution

$f_{R}(R)=\frac{R}{\epsilon^{2}} \exp \left(-\frac{R^{2}+2 \mu^{2}}{2 \epsilon^{2}}\right) I_{0}\left(\frac{\sqrt{2} R \mu}{\epsilon^{2}}\right), \quad R \geq 0$,

where $I_{0}(x)$ is zero-order modified Bessel function.

In Fig. 4, for the given parameter $\mu=0.1$, the contours of detection probability $P_{d}$ are plotted as a function of the noise level $\epsilon$ and the detector threshold $\lambda$. It is seen in Fig. 4 that there is a peak region around the non-zero noise level $\epsilon=0.18$, this is the stochastic resonance effect. For an arbitrary threshold $\lambda$, there is an optimal noise level $\epsilon$ that maximizes $P_{d}$. Here, the total signal power $n \theta^{2} P_{s}^{2}=1$ and the constant false alarm probability $P_{f}=10^{-3}$.

An important issue is that, for a given noise level, we can tune the threshold $\lambda$ to maximize the detection probability $P_{d}[1,8,11]$. Then, it is interesting to compare the maximum detection probability $P_{d}$ achieved by tuning noise level with that obtained by tuning threshold parameter $\lambda$. For instance in Fig. 4 , the red square marker in the plane $(\epsilon, \lambda)$ corresponds to the detection probability $P_{d}=0.47$ for the given noise level parameters $\epsilon=0.12$ and threshold parameter $\lambda=0.1$. There are two methods of further enhancing the detection probability $P_{d}$. Keeping noise level $\epsilon=0.12$, we can tune the threshold parameter $\lambda$ to 0.02 , and obtain the optimized $P_{d}=0.76$, as shown in Fig. 4 by the blue circle. However, it is shown in Fig. 4 that, we can also keep the detector threshold $\lambda=0.1$, and increase the noise level $\epsilon$ from 0.12 to 0.18 , leading to the optimal detection probability $P_{d}=0.90$ indicated by the downward green triangle. This comparison result demonstrate that the method of noise-enhanced detection is superior to the parameter tuning method in the considered cases. We note that this conclusion holds in the region of $\epsilon<0.15$ and $\lambda \leq 0.1$ for the Rice distribution, as shown in Fig. 4 divided by dashed green lines. However, this positive argument of noise is not observed for the generalized Rayleigh and Hall noise model.

\section{Conclusion}

In this paper, we explore non-Gaussian noise benefits to narrowband weak signal detection in an ad hoc hard-limiter detector.
Assuming the joint probability density of inphase and quadrature noise components is circularly symmetric, the noise-enhanced detection effects are demonstrated for the generalized Rayleigh, Hall and Rice noise models. Importantly, for the Rice noise model, the detectability can be improved by optimizing the threshold or the noise level. It is found that, under some conditions, the optimal detection probability achieved by the noise-enhanced method is better than that obtained by the method of tuning threshold. This interesting result indicates that, in some special cases, noise can actually play an alternative helpful part in improving the detector performance.

For low-pass weak signal detection, the performance of a locally optimum detector is closely tied to the quantity of Fisher information of noise distribution. Based on the Fisher information inequalities, we demonstrated that the addition of extra noise cannot improve locally optimal processing under the asymptotic conditions [15]. However, outside these restrictive conditions of weak signal and large sample size, improvement by addition of noise through stochastic resonance can be achieved, and becomes an attractive option for nonlinear signal processing. For the narrowband signal detection, the maximum efficacy of the locally optimum detector in Eq. (14) is not exactly the Fisher information of noise envelope distribution. Moreover, the addition of extra noise to the given narrowband signal will result in the joint probability density of inphase and quadrature noise components being not circularly symmetric. Therefore, there is an open question in narrowband signal detection, as to whether or not the addition of extra noise to the signal can improve the performance of a locally optimum detector. This question deserves to be further studied. Besides the considered hard-limiter detector having a very simple practical implementation, there are other available suboptimal detectors employed for narrowband signal detection, such as the soft-limiter detector [1], adaptive detector [4] and robust limiter-square detector [24]. Although such detectors are more complex for practical implementation, it is also interesting to verify the noise-enhanced effect in these detectors.

\section{References}

[1] S.A. Kassam, Signal Detection in Non-Gaussian Noise, Springer-Verlag, New York, 1988.

[2] J.W. Modestino, A.Y. Ningo, Detection of weak signals in narrowband nonGaussian noise, IEEE Trans. Inf. Theory 25 (1979) 592-600.

[3] N.H. Lu, B.A. Eisenstein, Detection of weak signals in non-Gaussian noise, IEEE Trans. Inf. Theory 27 (1981) 755-771.

[4] N.H. Lu, B.A. Eisenstein, Suboptimum detection of weak signals in non-Gaussian noise, IEEE Trans. Inf. Theory 29 (1983) 462-466.

[5] R. Benzi, A. Sutera, A. Vulpiani, The mechanism of stochastic resonance, J. Phys. A, Math. Gen. 14 (1981) L453-L457.

[6] K. Loerincz, Z. Gingl, L.B. Kish, A.R. Bulsara, Higher order stochastic resonance in a level-crossing detector, Phys. Lett. A 254 (1999) 154-157.

[7] X. Pei, K. Bachmann, F. Moss, The detection threshold, noise and stochastic resonance in the Fitzhugh-Nagumo neuron model, Phys. Lett. A 206 (1995) 61-65.

[8] P. Jung, Stochastic resonance and optimal design of threshold detectors, Phys. Lett. A 207 (1995) 93-104.

[9] S. Kay, Can detectability be improved by adding noise?, IEEE Signal Process. Lett. 7 (2000) 8-10.

[10] S. Zozor, P.O. Amblard, Stochastic resonance in locally optimal detectors, IEEE Trans. Signal Process. 51 (2003) 3177-3181.

[11] H. Chen, P.K. Varshney, S.M. Kay, J.H. Michels, Noise enhanced nonparametric detection, IEEE Trans. Inf. Theory 55 (2009) 499-506.

[12] R. Morse, A. Longtin, Coherence and stochastic resonance in threshold crossing detectors with delayed feedback, Phys. Lett. A 359 (2006) 640-646.

[13] F. Chapeau-Blondeau, D. Rousseau, Raising the noise to improve performance in optimal processing, J. Stat. Mech. Theory Exp. 1 (2009) P01003.

[14] A. Patel, B. Kosko, Noise benefits in quantizer-array correlation detection and watermark decoding, IEEE Trans. Signal Process. 59 (2011) 488-505.

[15] F. Duan, F. Chapeau-Blondeau, D. Abbott, Fisher information as a metric of locally optimal processing and stochastic resonance, PLoS ONE 7 (2012) e34282. 
[16] P.I. Fierens, S.A. Ibáñez, R.P.J. Perazzo, G.A. Patterson, D.F. Grosz, A memory device sustained by noise, Phys. Lett. A 374 (2010) 2207-2209.

[17] S. Barbay, G. Giacomelli, F. Marin, Noise-assisted transmission of binary information: Theory and experiment, Phys. Rev, E 63 (2001) 051110.

[18] G.A. Patterson, A.F. Goya, P.I. Fierens, S.A. Ibáñez, D.F. Grosz, Experimental investigation of noise-assisted information transmission and storage via stochastic resonance, Physica A 389 (2010) 1965-1970.

[19] N.G. Stocks, Information transmission in parallel threshold arrays: suprathreshold stochastic resonance, Phys. Rev. E 63 (2001) 041114.

[20] F. Duan, F. Chapeau-Blondeau, D. Abbott, Exploring weak-periodic-signal stochastic resonance in locally optimal processors with a Fisher information metric, Signal Process. 92 (2012) 3049-3055.
[21] S. Bayram, S. Gezici, Stochastic resonance in binary composite hypothesistesting problems in the Neyman-Pearson framework, Digit. Signal Process. 22 (2012) 391-406.

[22] V.N. Hari, G.V. Anand, A.B. Premkumar, A.S. Madhukumar, Design and performance analysis of a signal detector based on suprathreshold stochastic resonance, Signal Process. 92 (2012) 1745-1757.

[23] H.M. Hall, A New Model for 'Impulsive' Phenomena: Application to Atmospheric-Noise Communication Channels, Ph.D. dissertation, Stanford University, California, 1966.

[24] J.G. Shin, S.A. Kassam, Robust detector for narrow-band signals in non-Gaussian noise, J. Acoust. Soc. Am. 74 (1983) 527-533. 